\title{
Nicht jedes Immunsuppressivum ist bei allen Autoimmunerkrankungen wirksam
}

Fragestellung: Diese multizentrische randomisierte Doppelblindstudie untersuchte den steroidsparenden Effekt von Methotrexat in der Behandlung von Patienten mit Myasthenia gravis in einem Zeitraum von zwölf Monaten.

Hintergrund: Noch immer ist Azathioprin die einzig zugelassene steroidsparende medikamentöse Behandlung der Myasthenia gravis. Im klinischen Alltag werden jedoch in Analogie zu anderen Autoimmunerkrankungen auch andere Substanzen regelmäßig eingesetzt, selbst wenn für einzelne dieser Substanzen bislang keine überzeugenden Studiendaten vorliegen. Für die Behandlung mit Methotrexat konnte bisher nur eine nicht kontrollierte Studie eine zu Azathioprin vergleichbare Wirksamkeit zeigen.

Pasnoor M, He J, Herbelin L et al. A randomized controlled trial of methotrexate for patients with generalized myasthenia gravis. Neurology 2016; 87: 57-64
Patienten und Methodik: In pelblinden placebokontrollierten Studie wurden in den USA und Kanada 50 Patiendieser randomisierten, dop- ten mit einer generalisierten Myasthenie für zwölf Monate behandelt. Eine Gruppe erhielt Methotrexat und Steroide, während die andere Gruppe Steroide und Placebo erhielt. Die Steroiddosierung wurde nach klinischen Kriterien festgelegt. Methotrexat wurde nach einem Aufdosierungsschema mit $20 \mathrm{mg}$ pro Woche gegeben. Der primäre Endpunkt war die PrednisonDosis nach zwölf Monaten. Sekundäre Endpunkte waren klinische Scores und Parameter.

Ergebnisse: Die Studie hat ihren primären Endpunkt verfehlt. Nach zwölf Monaten hatte die Einnahme von Methotrexat zu keiner steroidsparenden Wirkung geführt. Auch die sekundären Parameter waren nicht signifikant und in den beiden Behandlungsarmen nicht unterschiedlich. Es traten keine schweren Nebenwirkungen auf.

Schlussfolgerung: Die Autoren folgern, dass Methotrexat in der Behandlung der Myasthenia gravis keine Wirkung hat, da sich nach zwölf Monaten kein Unterschied im Steroidbedarf in beiden Behandlungsarmen zeigte.

\section{- Kommentar von Tim Hagenacker, Essen}

\section{Wirkung von Immunsuppressiva nicht generalisieren}

Diese sauber durchgeführte Studie deckt gleich mehrere Probleme in der Behandlung neuroimmunologischer Erkrankungen auf. Methodisch räumen die Autoren ein, dass der Behandlungszeitraum mitunter zu kurz gewesen sein könnte. Außerdem werden Probleme in der Berechnung der Stichprobengröße in Erwägung gezogen, da der Steroidbedarf der Studienpatienten doch eine deutlich höhere Varianz zeigte, als ursprünglich angenommen. Aber selbst wenn sich nach Korrektur

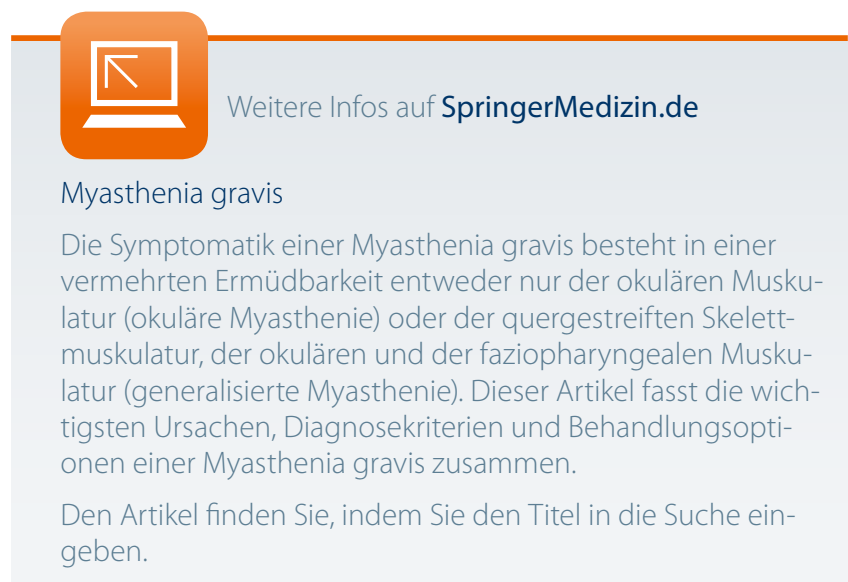

dieser Punkte signifikante Unterschiede zeigen sollten, wäre der Stellenwert dieser Effekte dann doch ohnehin eher zu vernachlässigen. Zum Nachdenken regt aber vor allem die Tatsache an, dass solch eine wichtige Studie erst nach so vielen Jahren der Behandlung neuromuskulär erkrankter Patienten durchgeführt wird. Dies bringt vor allem zum Ausdruck, dass wir uns nicht dem Gedanken hingeben sollten, die Wirksamkeit von Immunsuppressiva generalisiert auf alle autoimmunbedingten Erkrankungen unkritisch zu übertragen. Die Wirksamkeit von Methotrexat ist bei rheumatoiden Erkrankungen unbestritten, dort spielt aber auch der antiinflammatorische Effekt eine wichtige Rolle. Je mehr wir über die pathophysiologischen Mechanismen einer Erkrankung wissen, desto mehr müssen wir dieses Wissen in Therapiestudien umsetzen.

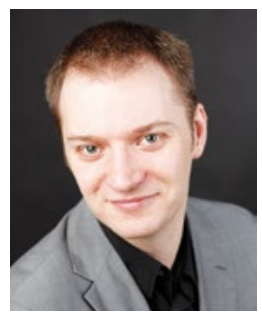

PD Dr. med. Tim Hagenacker, Essen

Klinik für Neurologie,

Universitätsklinikum Essen

E-Mail: tim.hagenacker@uk-essen.de 